\title{
Dissection of anti-inflammatory and immunomodulatory activity of Mangifera indica L. reveals the modulation of mPGES-1/PPAR $\gamma$ axis and Th17/Treg ratio.
}

\author{
Anella Saviano ${ }^{1}$, Federica Raucci ${ }^{1}$, Gian Casillo ${ }^{1}$, Adel Mansour ${ }^{2}$, Vincenzo Piccolo ${ }^{1}$, \\ Camilla Montesano ${ }^{3}$, Martina Smimmo ${ }^{1}$, Valentina Vellecco ${ }^{1}$, Gennaro Capasso ${ }^{4}$, Amedeo \\ Boscaino $^{4}$, Vincenzo Summa ${ }^{1}$, Nicola Mascolo ${ }^{1}$, Asif Iqbal ${ }^{2}$, Mariarosaria Bucci ${ }^{1}$, Giuseppe \\ Cirino $^{1}$, Vincenzo Brancaleone ${ }^{5}$, and Francesco Maione ${ }^{1}$ \\ ${ }^{1}$ University of Naples Federico II School of Medicine and Surgery \\ ${ }^{2}$ University of Birmingham College of Medical and Dental Sciences \\ ${ }^{3}$ University of Rome La Sapienza Faculty of Pharmacy and Medicine \\ ${ }^{4}$ Antonio Cardarelli Hospital \\ ${ }^{5}$ University of Basilicata
}

December 21, 2021

\begin{abstract}
Background and Purpose: In the context of inflammation and immunity, there are fragmented and observational studies relating to the pharmacological activity of Mangifera indica L. and its main active component mangiferin. We, therefore, aimed to evaluate the potential beneficial effects of this plant extract (MIE, $90 \%$ in mangiferin) in a mouse model of gouty arthritis, dissecting the cellular immune phenotypes and the biochemical mechanism/s beyond its activity. Experimental Approach: Gouty arthritis was induced by the intra-articular administration of MSU crystals (200 $\mu \mathrm{g} 20 \mu \mathrm{l}-1)$. MIE (0.1-10 mg kg-1) or corresponding vehicle (DMSO/saline 1:3) were orally administrated concomitantly to MSU (time 0), 6 and $12 \mathrm{~h}$ after the stimulus. Thereafter, knee joint score and oedema were evaluated in addition to western blot analysis for several components of mPGES-1/PPAR $r$ pathway. Moreover, the analysis of pro/anti-inflammatory cyto-chemokines coupled to the assessment of the cellular infiltrate's phenotype was investigated. Key Results: Treatment with MIE revealed a dose-dependent reduction in joint inflammatory scores with maximal inhibition observed at $10 \mathrm{mg} \mathrm{kg}-1$. MIE significantly reduced leukocyte infiltration and activation and the expression of different pro-inflammatory cyto-chemokines in inflamed tissues. Furthermore, biochemical analysis revealed that MIE modulated COX-2/mPGES-1 and mPGDS-1/PPAR $\curlyvee$ pathways. Flow cytometry analysis also highlighted a prominent modulation of infiltrating inflammatory monocytes (CD11b+ve/CD115+ve/LY6Chi), and (both infiltrated and circulating) Treg cells (CD4+ve/CD25+ve/FOXP3+ve) after MIE treatment. Conclusion and Implications: Collectively, the results of this study demonstrate a novel function of MIE to positively affect the local and systemic inflammatory/immunological perturbance in the onset and progression of gouty arthritis.
\end{abstract}

\section{Hosted file}

Saviano et al. Manuscript.doc available at https://authorea.com/users/452159/articles/550305dissection-of-anti-inflammatory-and-immunomodulatory-activity-of-mangifera-indica-1reveals-the-modulation-of-mpges-1-ppar\%CE\%B3-axis-and-th17-treg-ratio 
$\mathbf{A}$

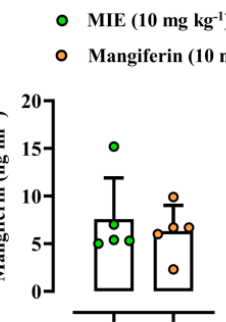

C

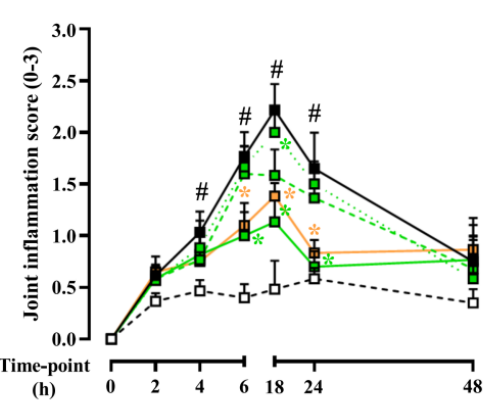

B $\quad$ MSU $\left(200 \mu \mathrm{g} 20 \mu^{-1}\right)$ i.a. Joint inflammation score

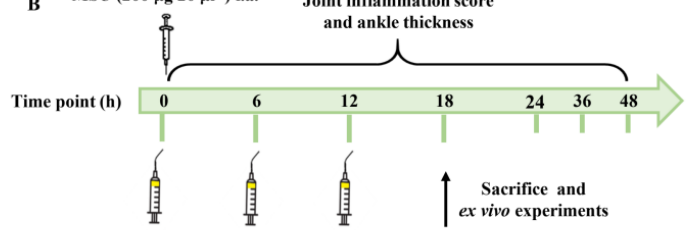

Mangifera indica extract $\longrightarrow \quad-\quad \longrightarrow$ Ctrl

$\rightarrow$ MSU $200 \mu \mathrm{g} 20 \mu \mathrm{l}^{-1}+$ MIE vehicle

ㅁ. MSU + MIE (0.1 $\left.\mathrm{mg} \mathrm{kg}^{-1}\right)$

- . $\mathrm{MSU}+\mathrm{MIE}\left(1 \mathrm{mg} \mathrm{kg}^{-1}\right)$

$\begin{array}{lll}D & & \\ & 1.50 & \text { MSU + MIE }\left(10 \mathrm{mg} \mathrm{kg}^{-1}\right) \\ & - & \text { MSU + Mangiferin }\left(10 \mathrm{mg} \mathrm{kg}^{-1}\right)\end{array}$

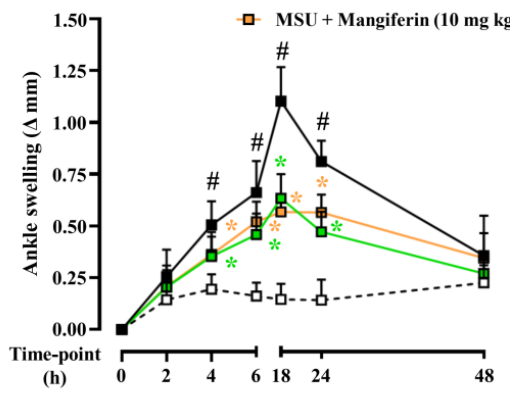

E

MSU + MIE

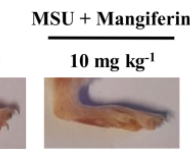

G

- MIE

F
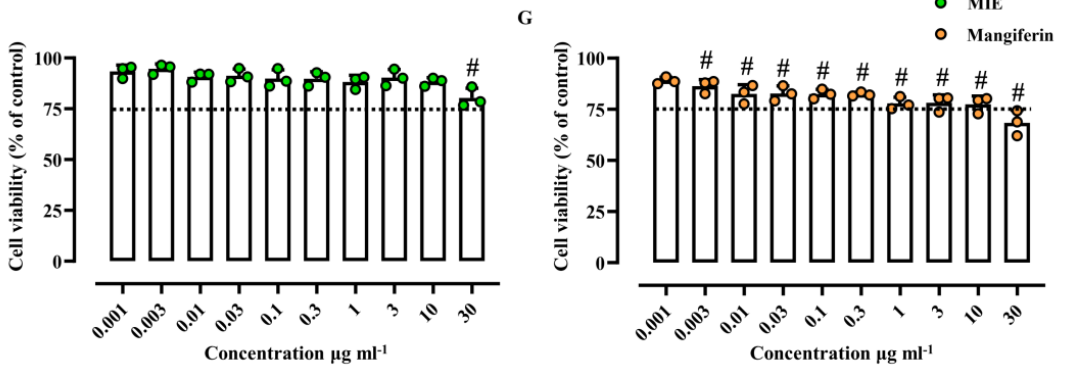

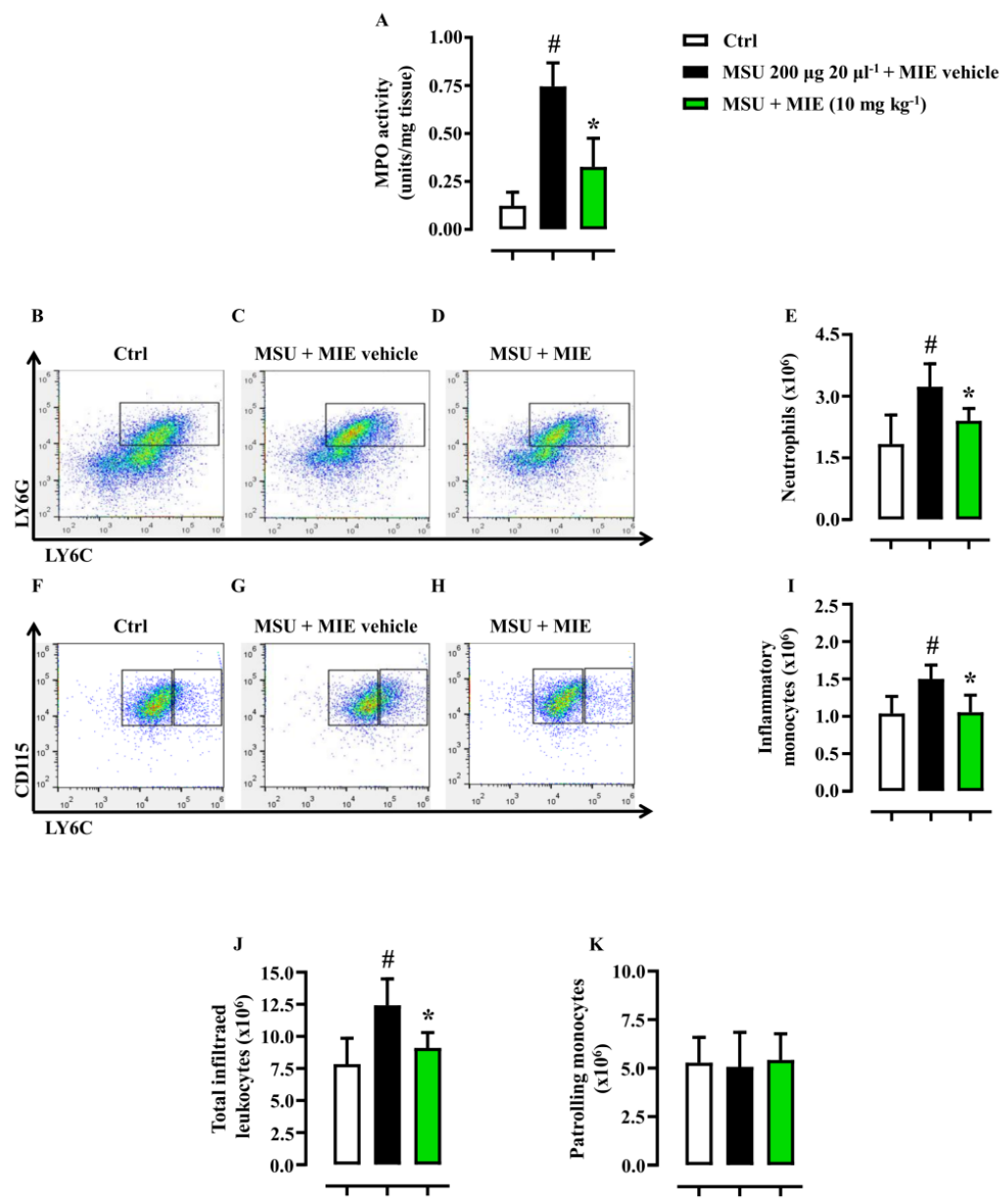

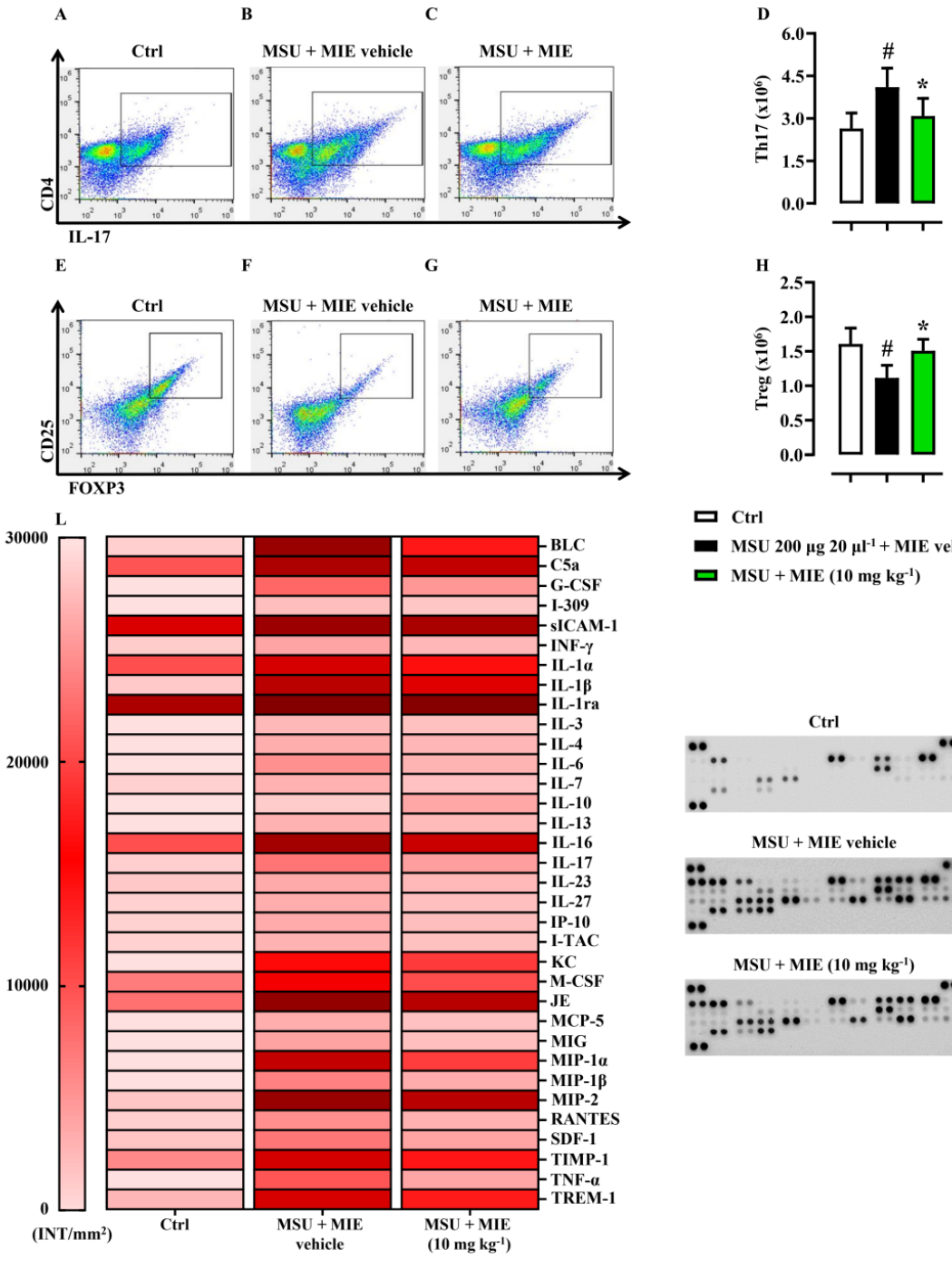

ロ Ctrl

- MSU $200 \mu \mathrm{g} 20 \mathrm{\mu l}^{-1}+$ MIE vehicle

口 MSU + MIE (10 $\mathrm{mg} \mathrm{kg}^{-1}$ )

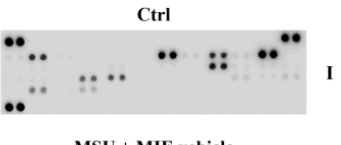

MSU + MIE vehicle

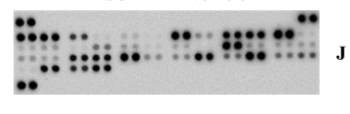

MSU + MIE (10 $\left.\mathrm{mg} \mathrm{kg}^{-1}\right)$

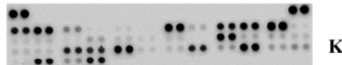

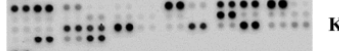




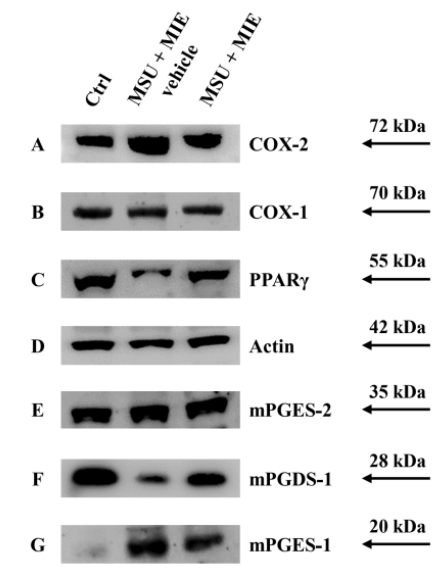

H

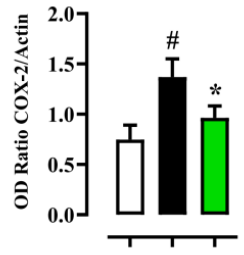

K

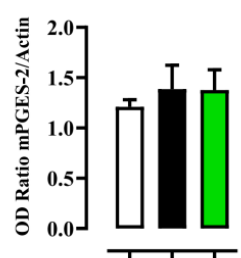

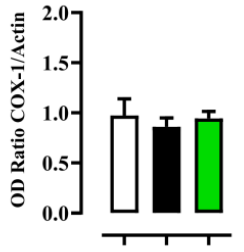

L

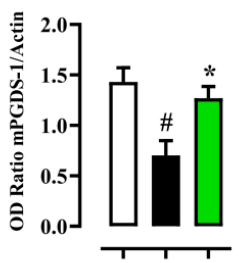

ロ Ctrl

- MSU $200 \mu \mathrm{g} 20 \mu \mathrm{l}^{-1}+$ MIE vehicle

口 MSU + MIE (10 $\left.\mathrm{g} \mathrm{kg}^{-1}\right)$

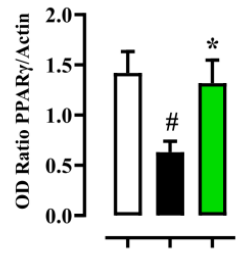

M

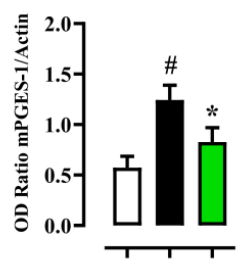



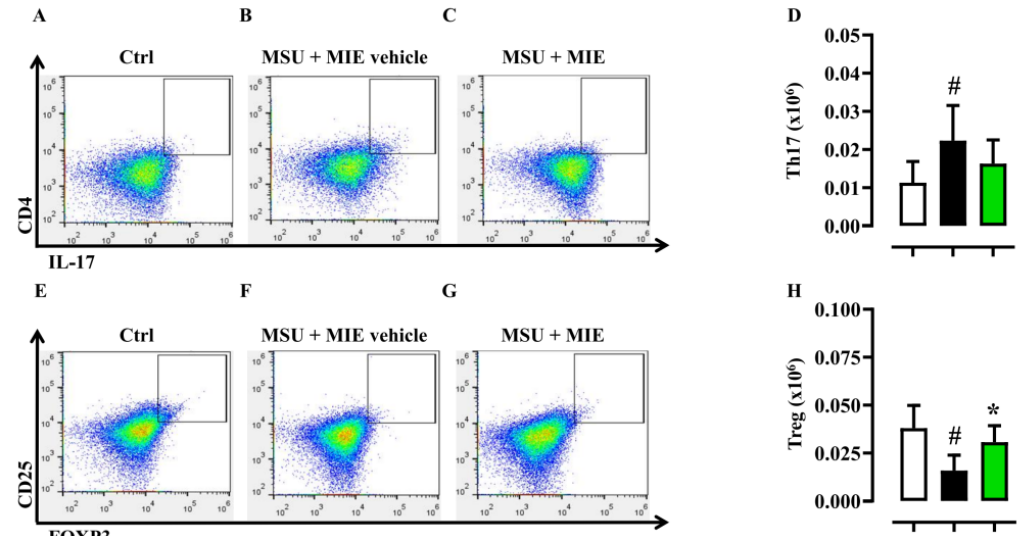

H
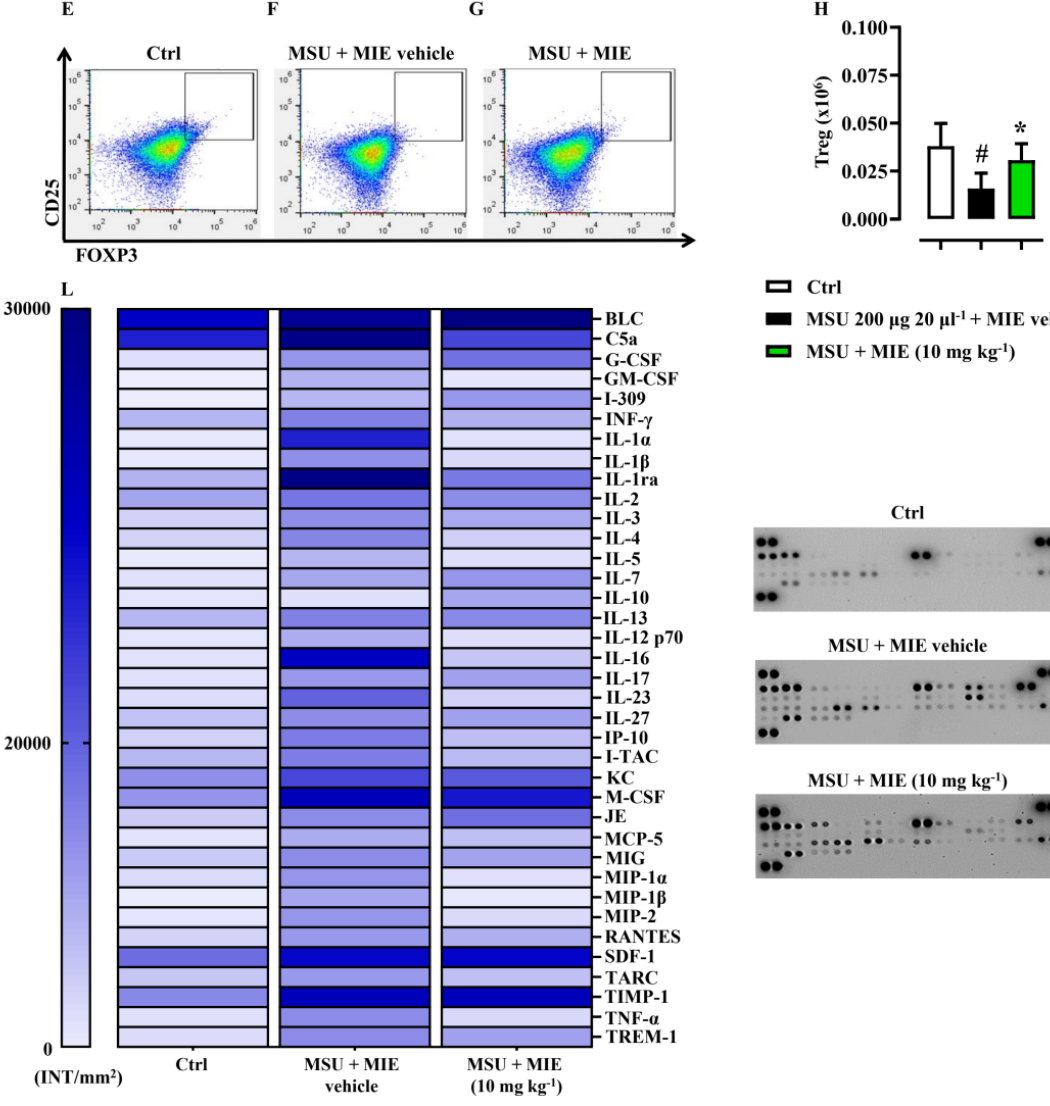

口 Ctrl

- MSU $200 \mu \mathrm{g} 20 \mathrm{\mu l}^{-1}+$ MIE vehicle

口 MSU + MIE (10 $\left.\mathrm{mg} \mathrm{kg}^{-1}\right)$

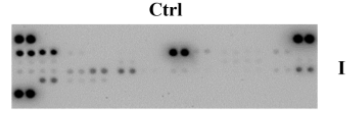

MSU + MIE vehicle

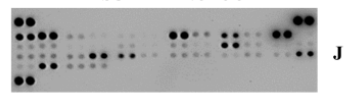

MSU + MIE (10 $\left.\mathrm{mg} \mathrm{kg}^{-1}\right)$

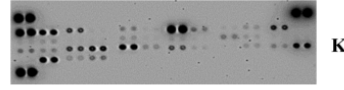




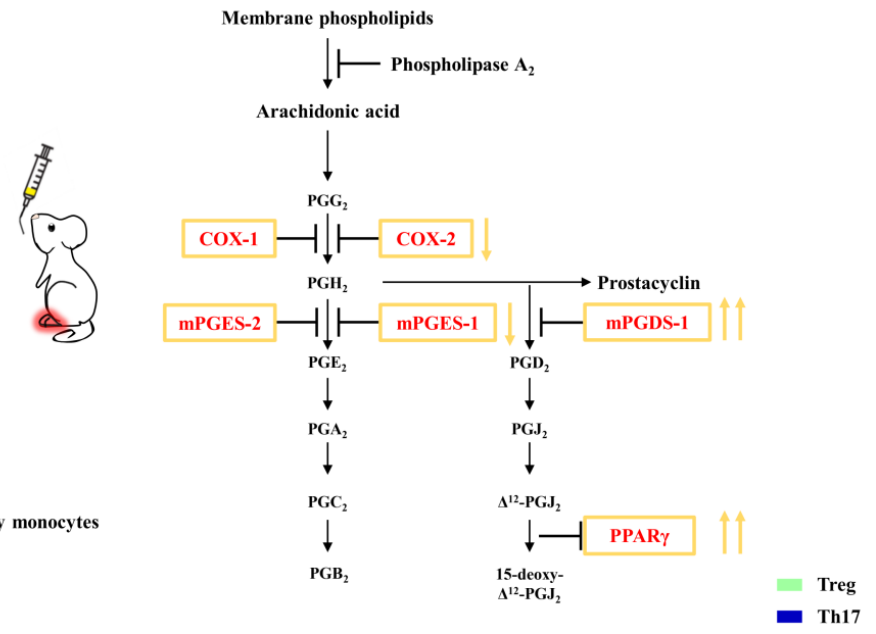

- Inflammatory

$$
\text { - Treg }
$$$$
\text { - Th17 }
$$

B

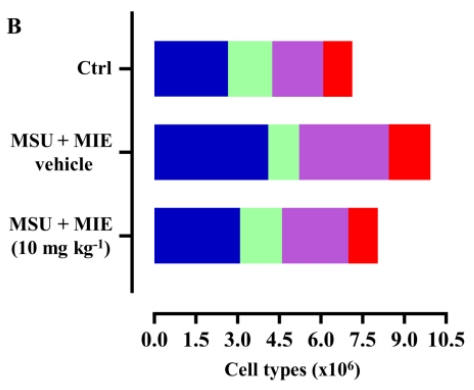

D

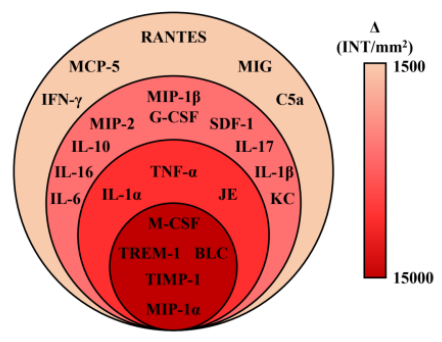

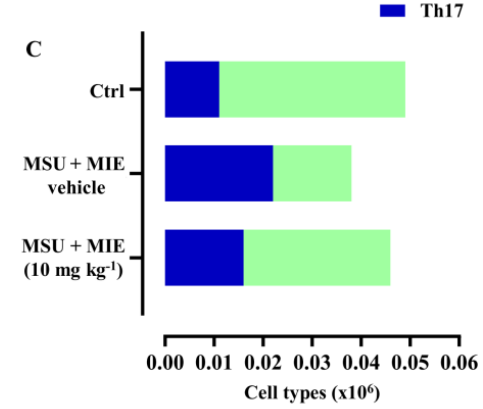

E

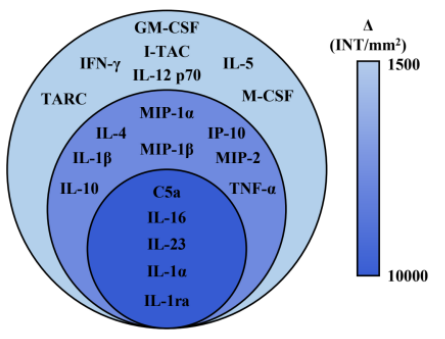

